\title{
No Data, No Problem, No Action: Addressing Urban Health Inequity in the 21st Century
}

\author{
Sharon Friel, David Vlahov, and Robert M. Buckley
}

In all countries, rich and poor, the move to urban living has been both good and bad for health and has contributed to health inequities within countries (the urban-rural divide) and within cities (the social gradient). The health risks associated with accelerating urbanization in the context of other global challenges, such as climate change, food insecurity, and financial crises-especially in countries with low levels of infrastructure and health system preparedness-pose significant development challenges in the 21st century.

But urbanization does not have to be negative. There is considerable global recognition that the attention to the way in which urbanization takes place provides a major opportunity not only to help economic development, but also local, national, and global health, and at the same time to address environmental concerns. Many of the authors involved in this series have written about what is required to improve urban health and health equity. A synopsis of those writings would read along the lines of "communities and cities that ensure access to basic goods such as sanitation, education, health care and social services, that are designed in ways that build social cohesion and promote good physical and psychological well-being, and that are protective of the natural environment are essential for health equity.”

While today we can describe many of the features of a healthy and sustainable city, and the governance and planning processes needed to achieve these ends, there is still much to learn, especially with respect to applying these concepts in cities of low- and middle-income countries. Recognizing this, the Rockefeller Foundation provided funding for two sister projects in 2009/2010, Global Research Network for Urban Health Equity (GRNUHE) and Roundtable on Urban Living Environment Research (RULER). Each convened a network of urban experts from a variety of disciplines, sectors, and continents.

GRNUHE reviewed the current knowledge in the social and environmental determinants of urban health inequities, documented what is and is not working to improve urban health equity, and identified gaps in the evidence base, thereby setting a direction for a global research agenda. All of these processes of governance, the various aspects of urban life and their outcomes in terms of health and health inequity need to be measured and monitored so that policy and program development, evaluation, and accountability are possible. It was these very issues that were

Friel is with the The National Centre for Epidemiology and Population Health, The Australian National University, Canberra, Australia; Vlahov is with the University of California, San Francisco School of Nursing, San Francisco, CA, USA; Buckley is with the RockefellerFoundation, New York, NY, USA.

Correspondence: Sharon Friel, The National Centre for Epidemiology and Population Health, The Australian National University, Mills Road, Canberra, ACT 0200, Australia. (E-mail: Sharon.Friel@anu. edu.au) 
of interest to RULER. RULER reviewed existing methods of measurement for urban health, aiming to identify gaps in measurement that must be filled in order to assess and evaluate population health and health equity in urban settings, especially in informal settlements (or slums) in low- and middle-income countries.

In this series of papers, we report the main findings from GRNUHE and RULER. GRNUHE demonstrates how urban planning and design and urban social conditions can be good or bad for health equity depending on how they are set up, and shows some promising planning and social policies and practices from around the world. We describe how climate change mitigation and adaptation can to go hand-in-hand with efforts to achieve health equity through action in the social determinants. Drawing on examples from all continents, we highlight how different forms of governance can shape agendas, policies, and programs in ways that are inclusive and health promoting, or can perpetuate social exclusion, inequitable distribution of resources, and associated inequities in health. By outlining an integrated applied research agenda, we aim to assist researchers, policy-makers, service providers, and funding bodies/donors to better support, coordinate, and undertake action that is organized around a conceptual framework that positions health, equity, and sustainability as a central policy goal for urban management.

RULER describes how existing measurement systems focus primarily on health outcomes and systems, mainly at the national level. Although substantial reviews of health outcomes and health service measures had been conducted elsewhere, such reviews covered these in an aggregate way. Relatively few countries have examined their inter- or intra-urban health inequities and even fewer do so on a regular basis. RULER observed that some urban health metrics were already available, if not always appreciated and utilized in ongoing efforts (e.g., census data with granular data on households, water, and sanitation but with little attention paid to the spatial dimensions of these data). However, in general there is a paucity of evidence on the social determinants of health and health inequities at the city level. Other, less obvious elements had not exploited the gains realized in spatial measurement technology and techniques (e.g., defining geographic and social urban informal settlement boundaries, classification of population-based amenities and hazards, and innovative spatial measurement of local governance for health). In summary, the RULER team identified three major areas for enhancing measurement to motivate action for urban health, namely, disaggregation of geographic areas for intra-urban risk assessment and action; measures for both social environment and governance; and measures for a better understanding of the implications of the physical (e.g., climate) and built environments for health. The challenge of addressing these elements in resource-poor settings was acknowledged, as was the intensely political nature of urban health metrics. Also, overcoming these challenges will be expensive, far too expensive for foundations alone. This is unambiguously a problem and is the sort of task that global health agencies such as the United Nations should systematically address.

Urban development that places health equity as a central policy goal will improve health, reduce social inequity, and support communities to cope with, and avert further, global environmental change. To do this requires fine-grained measurement, multilevel monitoring systems, action on the social and environmental determinants of health and inclusive systems of governance. 